\title{
Affective Syndromes
}

\author{
Parvaneh Tirsahar* \\ University of Tutor, UK \\ Submission: October 26, 2016; Published: January 09, 2017 \\ *Corresponding author: Parvaneh Tirsahar, University of Tutor, London; UK, Email: paria.kiarash@yahoo.com
}

Abbreviations: CBT: Cognitive Behavioral Therapy; PTSD: Post Traumatic Stress Disorder; AD: Anti-depressive Drugs; IPT: Interpersonal Psycho-Therapy; DSM-IV: Diagnostic Statistical Manual of Mental Disorders - Fourth Edition

\section{Introduction}

Some people entertain a very bright outlook on life and radiate a strongly contagious zest and appetite for life. They are foreign to sorrow and greet the day with a smile. This has very little to do with mania which indicates a pathologically elevated level of activity. This is a disease that is caused by a disruption of the basic mood level and may be secondary to other disorders, or it may be the primary disorder. Manic depression is an affective syndrome and features two distinct phases: one of depression and one of hy be periods with no symptoms at all [1] (Figure 1).

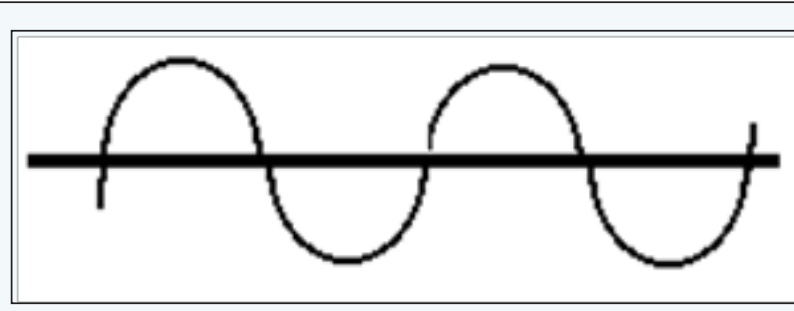

Figure 1: Affective Syndromes.

From a theoretical standpoint I have set out to show what an affective syndrome is, re garding both definition and treatment. My objective for this study is to enhance understanding of the phenomenon of manic depression as well as making a comparison of various methods of treatment that are in use by performing interviews with ward staff. The results show that lithium yields best results and that the diagnostics is based upon the various symptoms that are typical of the disease.

My interest in studying manic depression derives partly from personal experiences of working as nurse and also because more knowledge among the public on this subject would be desirable. According to Ottosson (2000) [2], altered basic mood is often secondary to schizo $\neg$ phrenia, dementia, alcoholism, personality disorders, and other psychological disorders. Variาous adverse life experiences as well as struggles may lead to affective syndromes but are not sufficient nor is the outcome inevitable, meaning that affective syndromes may surface nev?ertheless. Psychological trauma may cause psychological disorders and are treated under the heading of crisis reactions, post-traumatic stress syndromes, or adaptive disorders.

There are similarities between bipolar disease and postnatal depression. Half of the symp ᄀtoms in both bipolar disease and postnatal depression are maniacal and the other half are de $\neg$ pressive [3]. An individual with bipolar disease shows powerful mood swings that are stronger than or $\urcorner$ dinary ups and downs. The most typical characteristics of the syndrome are alternating epi $\neg$ sodes of lowered mood (depression) followed by elevated mood (mania). The patient may be suffering from delusions and may sometimes be separated from reality.

As stated earlier, an individual usually functions normally between these phases. About one percent of the population in the US and Sweden suffer from bipolar disease [2,4]. Hereditary factors and altered functionality of neurotransmitters in the brain, such as serotonin and dopamine, play an important part in the development of bipolar disease [2]. According to Skärsäter (2002) [5], a major depression will have an impact upon affective, cognitive, behavioural, social, and physiological functions. In mixed or agitated depression the patient is restless and shows stereotypical and repeti $\neg$ tive movements that are an expression of internal disquiet. In mixed depression, at least two of the following symptoms must be present:
a. Internal conflict
b. Psychomotor irritability
c. Topical flight [6] 
Apart from showing irritability, children with mania can be extremely moody experiencing explosive episodes that last for an hour or longer. During such explosive episodes children may do things such as damaging other people's property or household equipment or making holes in the wall. It may be difficult to calm down such fits of rage when the child is swearing and being very hostile [7]. Bipolar disorder among children and adults are difficult to distinguish from age related problems. For instance, irritability and aggression associated to bipolar disease may also be caused by attention deficit hyper-activity disorder, conduct disorder, oppositional defiant disorder, depression, or schizophrenia. Drug abuse may also lead to such symptoms [4].

Hereditary factors play an important part in the development of bipolar disease. The func $\neg$ tion of major neurotransmitters (serotonin, noradrenalin, and dopamine) is also altered. How $\urcorner$ ever, a study from the University of Taipei showed that the connection between bipolar dis $\neg$ ease and X-box proteins is unclear [8]. The doctor will make the diagnosis based upon the various symptoms of the disease. Just as with other psychiatric diseases there is no blood sample or brain scanning to do; the diag $\neg$ nosis is made on the symptoms that are present. One may also look for similar cases among relatives. Despite a great risk of relapse, fortunately there are effective treatments available such as, for example, preventive medicine.

\section{Treatment}

The kind of treatment chosen is determined by the severity of the symptoms. Conversaᄀtional therapy may work with light depressions. But with cases of mania and severe depressions medication is required. It has been proven that lithium and antileptic drugs have a modifying effect on mood [9]. Individuals with least social functional disorders respond best to interpersonal psychother $a$ apy while those with least cognitive dysfunctions responded best to cognitive behavioural therapy (the least severe cases responded best). Individuals with reduced working capability as well as those with the heaviest symptoms responded best to imipramine [10]. Cognitive behavioural therapy (CBT) is a form of pedagogical psychotherapy based on a sup $\neg$ position that cognitive factors - ways of thinking about oneself, the environment and the furture - influence mental conditions, and that altering one's thoughts may have positive effects upon mood [9].

The purpose of this study is increasing understanding of the manic-depressive phenomenon as well as discussing existing diagnostics and forms of treatment for manic depression. The objective of the following theoretical is presenting different perspectives of the phenomenon. These perspectives pose the following queries: How is bipolar disease expressed? How does the doctor go about making the diagnosis? How is bipolar disease treated? Which treat $\neg$ ment is the most effective? What can the patient do? Both depression and mania are conditions known since antiquity as is the fact that some people swing between them. The word melancholia means "black bile" (from the Greek words melas which means black, and khole which means bile), and is a residue from Galenos's theory of bodily fluids and their balance. Emil Kraepelin determined 1896 that mania and melancholia are different phases of one and the same disease, manic-melancholic psychosis, and differs from schizophrenia, both in the sense that it is episodic with full recovery between phases, and because of its longevity and symptoms [11].

Emotions (feelings) are divided into affects and basic moods. Affects are responses to life situations that are of relatively short duration while moods are more durable and seldom con $\neg$ nected to outer circumstances. For instance, if you are down a few days after a failure, that would be regarded as an effect, whereas an annually recurring spring depression means dis $\neg$ ruption of the basic mood. The most important mark of an affective syndrome is altered basic mood. In English literature this is alternatively called affective or mood disorders. Swedish and other studies abroad have shown that the chance during a life time of suffering from an affective syndrome is $15-27 \%$ among men and $20-45 \%$ among women. By the same token, the risk of suffering from an affective syndrome of psychotic nature is about $2 \%$ among men and $5 \%$ among women.

This increases with age. Altered basic mood, usually depression, may be secondary to schizophrenia, dementia, anxiety syndrome, alcoholism, personality disorders among several other mental disorders. Both international and American classification maintains a distinction between strong euphoria (mania) and low mood (depression). Both classi $\neg$ fications also distinguish between occasional episodes in time from episodes that are recur $\neg$ ring. Such periods may be unipolar, i.e., swinging up and returning to normal (or as the case may be, swinging downwards and returning to normal), or they may be bipolar, i.e., sometimes swinging up and sometimes down. There are even labels for long term phases like dysthymia (gloom, low mood) and cyclothymia (a sort of lighter version of manic depression); however, hyperthy $\neg$ mia is not the opposite of dysthymia [2].

Thyroid is the hormone that stabilises mood. Bipolar patients usually have thyroid disruptions. Too much or too little thyroid hormone in the blood stream leads to mood and energy changes. High thyroid levels should be treated with thyroid hormone tablets as well as with medication against bipolar disease [4]. Diseases like PTSD (Post Traumatic Stress Disorder), stress, obsessions and compulsory disorders are common with maniacal depressive patients as well as alcoholism and drug abuse [4]. There are similarities between bipolar disease and post natal syndromes. I both bipolar disease and post natal syndromes, half of the symptoms are maniacal and the other are depressive. In both bipolar disease and schizophrenia there are maniacal and depressive symptoms [3].

Ottosson (2000) [2] holds that various adverse events and challenges in life lead to affective syndromes although they are neither necessary nor sufficient, i.e., affective syndromes may surface nevertheless. Psychiatrically traumas cause mental 
disorders that are catalogued under headings such as crisis reactions, post-traumatic stress syndromes and adaptive disorders. The main categories of affective episodes are of maniacal and depressive character. There is also a so called mixed condition where depressive and maniacal symptoms may coexist or alternate. Cycloid episodes (a type of mental disorder that is a combination of affective syndromes and schizophrenia) on the other hand, are episodes in connection to psychotic symptoms that do not resonate with the basic mood level. One such episode may have a duration of at least two weeks although shorter duration may suffice if the symptoms are severe and the onset demands urgent response. A depressive episode may show different levels such as light, moderate or severe, depending on quantity, category or severity of the symptoms. A major depres $\neg$ sion may also show different severity levels.

According to IDC-10 a true depressive episode features the following:

a. Low mood

b. Decreased attention to all or nearly all work tasks, decreased desires or joy

c. Lack of energy or increased fatigue. Loss of self-esteem and self-awareness

d. Exhaustion or lack of energy

e. Absurd self-accusations or exaggerated and unfounded feelings of guilt

f. Regular thoughts about death or suicide

g. Loss of capability to think, cannot concentrate nor make decisions

h. Excitement or inhibitions and sleeping disorders

i. Significant loss of appetite or loss of weight.

According to Skärsäter (2002) [5], a major depression will have an impact upon affective, cogni $\neg$ tive, behavioural, social, and physiological functions. The cause is multi-factorial, sometimes of biological origin or sometimes of genetically origin, and sometimes it may be caused by an interaction of psychological and psycho-social stress. A major chronic depression may occa $\neg$ sionally last up to two years.

At least three of the following criteria must be met before a doctor can make a diagnosis:

i. Distinct depressive quality that is not the same as for instance grief at the loss of a loved one

ii. Sense of gloom that is worst in the morning

iii. Waking early, that the patient wakes up at least two hours earlier than usual

iv. Express psychomotor inhibition or agitation

v. Significant loss of appetite or weight loss vi. Exaggerated or unfounded feelings of guilt that have no real basis

Concerning mixed depression, at least two of the following symptoms must be present:

a. Inner conflict

b. Psychomotor irritability

c. Topical flight [6].

\section{Diagnosis}

The doctor makes the diagnosis based upon the different symptoms that are present. Just like the case with other mental diseases there are no blood samples to take, no brain scans to be performed; the diagnosis is solely based upon the symptoms that are present. About the first episode there may be uncertainties, but looking for other cases within the family may often prove helpful [12]. The general picture, however, with its extreme mood swings, is so characteristic that there seldom are any reasons to doubt the accuracy of the diagnosis.

Although a deeply depressed patient may experience sadness and a sense of abandonment and unhappiness, when interviewed he is not able to weep and there are no tears. There are differences between grief and depression that are difficult to describe. One obvious difference is that grief has a cause whereas the origins of depression are hard to pin down. Other characteristic traits of depression are condescending of self, feelings of guilt, hopelessness and meaninglessness. Sometimes a depression is affected by irritability and dysphoria. In less severe cases the self-depreciation is interpreted as lowered self-esteem and loss of inner security and power. One feels insecure before people with whom one once used to be close without any problems. Feelings of inadequacy, insignificance, unworthiness and misery may also be present. Feelings of guilt are an expression of an extended super-ego, conscience.

That is not the same as the feelings of guilt that follow fatigue and dependence and are common with mental disorders in general. The feelings of guilt that come with depression have an origin from actions that happened or did not happen a long time ago. For a religious person this is called sin, and for others childish guilt complexes may be triggered, about for instance masturbation, disobedience or dishonesty. Thoughts about guilt and punishment as well as self-depreciation of ethical, religious, body related or economical nature are symbolic of delusions. Hopelessness and meaninglessness are clear signs of depression. A deeply depressed individual does not expect anything positive from the future and does not ever expect any recovery. One measure of the depth of the depression is thoughts about death, such as death wishes, suicidal thoughts, intentions of committing suicide, and suicide attempts [2].

Working as a children's analyst Melanie Klein offered a ground breaking contribution to the view on affective disorders. 
She claimed that children as early as their first year after birth had rich inner imagination where battles of love and hate play out in a dramatic way. The new-born child's fear of death and destruction is connected to strong emotions that are evoked by disappointment. Children have, Melanie Klein assumes, an innate cognitive capability to register and express not only one nurturing person but two, mother and father, and their relation to each other. The child struggles to protect itself by subdividing existence into good and bad experiences. Aggressive and hateful impulses create fantasies about persecution from the part of the object. All early object relations, from the oral phase through the anal and the urethral phases will be infused by the battle of good and evil. At one point in development the child will attempt to integrate these opposite tendencies. Klein calls this the depressive position. She believed that the child at this point in development is exposed to depressive affects caused by fear of liberating or losing the object of love [11].

Inhibition is a general word for symptoms like fatigue and absence of grace, strength and nuance. All mental functions are disturbed. Depending upon which functions are most affected there are different kinds of inhibition. Thinking proceeds slowly. The patient may find it difficult to concentrate and memory is unreliable. One feels stupid and incompetent. Ideas just disappear. Speech is slow and devoid of association, the replies consist of one syllable - this is called cognitive inhibition. One ruminates following to fixed thought patterns. Emotional inhibition is the case when the patients are placed under a veil of casualness (apathy) and lack of desire (anhedonia). Nothing is fun anymore, and the environment is registered in a dull, artificial and soulless way. One loses the affection for loved ones.

A deeply depressed individual looks ten years older than his years. To determine whether there is a motor inhibition the clinician may check muscle movements about the eyes. The extreme case is depressive stupor (complete immovability, total mental blockage) with almost complete motor inhibition. Cases where the patient's perception of time is disturbed must be classified as an inhibition symptom. Days, and particularly nights, are perceives as eternities. There is a difference between subjective and objective time which normally proceed the about same rate. Anxiety, disturbed vitality, anorexia, hypo salivation, reduced tear secretion, sleep disturbance, disturbed circadian rhythm and absence of reactivity is typical of depression. Anxiety in connection to depression may cause motor unrest - excitement - which may be present simultaneously or alternating with psychomotor blockage.

A maniacally inhibited person may, for instance, contin $\neg$ ually change position on a chair or walk restlessly to and fro in the room. In the depressive syndrome uneasiness may have several causes, such as feelings of guilt, hopelessness, meaninglessness, and a pressing feeling in the chest above the belly. Anxiety may be a reaction to procrastination, hypochondria, sexual impotence, or uneasiness upon early waking, etc. Bodily symptoms may be vague such as weakness, powerlessness, shivering, general feelings of illness, or limited to the chest, belly, or head area as a sense of pressure, gloom och tightness. It may feel like a severe hangover or a bad flu where the patient has lost any hope of feeling better anytime soon. Disturbances of central functions are often present also, such as those of sleep, appetite, eating, or sexuality. Whenever these symptoms are determined one speaks of vital depression which is synonymous with melancholic depression.

Other symptoms may also be present such as loss of appetite partly due to incapability to enjoy life and/or disturbances of belly and bowel functions. Loss of appetite is expressed in a way that renders tasteless and uninteresting items like food and drink that normally would evoke desire. Gaining weight is more frequent among young depressed individuals who eat lots of food (hyperphagia). Reduced saliva secretion is typical of depressive and maniacal syndromes. It is experienced as dryness of mouth and throat, elevated thirst and bad taste in the mouth, "acid break". Constipation is a consequence of low tonus and slowed down bowel movements leading to extended passage time [2].

The patient is not able to weep and the secretion of tears is reduced. Sexual activity is reduced or completely absent, i.e. the patient suffers from sexual reactivity. Among women even irregular periods or total absence of the period is found. These symptoms are found in connection to unchanging conditions and are therefore not mentally induced.

Early awakening is the typical sleep disorder found in depression. Sleep is not delayed but easily becomes disturbed and superficial. The patient wakes up frequently and finds it difficult to go back to sleep, and the final awakening happens earlier than normal and is accompanied with discontent and troubles, and occasionally with anxiety. Early awakening means waking up two hours early compared to normal. The day is experienced as very long and boring. Among younger depressed individuals hypersomnia is common. The day begins about three to four a.m. in connection with early awakening. The morning is cumbersome - this is the time when most suicides happen however, by the noon the troubles begin to fade and during the afternoon and evening life may seem reasonably good [2].

Ottosson (2000) [2] Also states that the vital symptoms are connected to a disorder of the hypothalamus. The hypothalamus is a supervising centre for control of biological pace, desires and other functions. The hypothalamus, in turn, is controlled by the autonomous nervous system and the pituitary gland. Depressive syndromes, especially those that are deep, cannot be manipulated in a positive direction. People who are affected cannot appreciate the joys of life, like for instance an entertaining movie, a visit by friends, or going somewhere on vacation. Everything is just painful and reminds the patient that he is not capable of enjoying life or being like everybody else. This nonreactivity, which sometimes is called massiveness or autonomy, also prevents the patient from reacting in a normal way upon negative events, such as the death of a loved one. 
Furthermore, Ottosson (2000) [2] writes that melancholic symptoms may have genetic, periodic, biochemical, neurophysiological, or hormonal origins, and may also be the result of somatic anti-depressive treatment (psychotropic drugs, ECT, light therapy, or waking therapy). The melancholically properties that are the most connected are incapability to enjoy the good things in life, absence of reactivity, a state of strong tensions, unease and restlessness, and varying circadian rhythm, and also inhibition and vital symptoms. In general, the clinical picture of depression among old people resembles that of young people. The only difference is that synthymous delusions are more common among older people and may show even when the basic mood level is not significantly lowered. This is sorted under the heading of pseudo dementia. Depressive syndromes among older people are often confused with progressive dementia and therefore left without adequate therapy treatment. A so called swimmers view of brain damage does not have to be manifest with dementia, and in case it is, that does not exclude simultaneous depression. Medical history, earlier affective episodes, positive family anamnesis and normal EEG would suggest depression. In cases that are not decisive the patient is offered psychotropic drugs or ECT.

Even among children or young people depressive syndromes may be atypical. Episodes of mania and depression may manifest from about ten years of age. Concentration difficulties, passivity, restlessness, aversion against school work, school phobia, or declining achievements are often unrecognised depressive sympᄀtoms. Some children experience hypochondriacal problems and believe themselves to be affected by brain tumours or leukaemia. Obstinacy, disobedience, fits of rage, destructivity, running away, and loose sexual liaisons sometimes prove to be mania equivalents [7]. I addition, Ottosson (2000) [2] states that the most common suicidal actions happen to depressed patients, and in particular melancholic patients. The risk of sui $\neg$ cide is greatest during the initiatory and concluding stages of a depression period, when inhibition is not as hidden as it is when the depression is at its deepest stages.

Experience shows that suicide also happens in the middle of an episode as an expression for a sudden breakthrough of anxiety (ruptus). Melancholically depressed patients sometimes plan suicide where they take their loved ones with them when they die. The diagnostics of an depression episode recognises that the first manifestation does not seem to make sense based upon psycho-social conditions, that depressive episodes will alternate with independent intervals without any evident connections to other events in life, that the family's medical history is positive, and that there is a spontaneous circadian variation.

Individuals that develop maniacal conditions or deep depression today are still offered medicinal treatment, such as neuroleptics for mania or anti-depressive drugs for deep depression. In those situations people will usually be admitted to hospital, either voluntarily or according to rules on compulsory treatment. The objective of these measures can be presented in the following points:

i. It is usually necessary to perform the treatment which the patient refuses (mania), or does not believe in (depression).

ii. The priority is to prevent a potential suicide as well as to apprehend the individual in order to prevent any injury that may follow the condition.

iii. The hospital environment can create the framework for psycho-social treatment forms, from simpler measures such as elucidating stress factors and dynamical conditions within the family, to more complex actions such as psychoanalytical oriented psychotherapy.

ECT is often used in many countries for treatment of deep depressions. Psychotherapy is another option. In recent years lithium treatment has become an established method, not least for preventive purposes. The effect on clear cases of maniacal depressive conditions is well documented. In many places in Denmark, Britain, and the US, lithium is recommended for less typical cases of mood disorders. However, both patient and doctor must recognise the risks of certain physiological damage, in particular concerning metabolic disruptions which may necessitate long term thyroxin treatment. There is also a great risk of birth defects when lithium is used during pregnancy. And finally, lithium treatment may be hazardous if administered when liver and kidney functions are impaired [11].

Ottosson (2000) [2] also maintains that anti-depressive treatment should be classified under two headings: Somatic (psychotropic drugs, sleep deprivation, light therapy) and psycho-social (psychotherapy, social measures). Choice of therapy is determined by the severity of the de 7 pression, clinical picture, aetiology, and procedure. Somatic therapy is motivated when the depression has melancholic or hereditary origin. Psychotherapy is recommended when the depression have originated from an interaction of events and character traits. The most severe depressions demand somatic treatment irrespective of aetiology, at least initially. Anti-depressive drugs (AD) are imipramine and the so called tricyclic AD drugs.

\section{Results}

According to Ottosson (2000) [2] many have noticed that absence of one night's sleep can produce strong euphoria next day. That is the basis for waking therapy which can be performed with total or selective (REM) deprivation. Waking therapy has become a standard procedure and used during the small hours of the night. It has greater effect on melancholic depression than other depressions. The drawback is that the effects are short-lived and relapses are common, as early as the second day after waking therapy; that is, after only one night's undisturbed sleep. Even a short nap may cause a relapse. This may partly be prevented by repeated waking therapy or a combination with $\mathrm{AD}$ or ECT, 
Light therapy is an option for depressions that are connected to the dark season (winter). The patient receives a quantum of light, about 6,000 to 10,000 lux for about two hours daily which usually will produce improvement within three days. When the treatment is terminated the improvement will decline as rapidly. For this reason the light therapy must continue until nat $\neg$ ural light becomes adequately bright [2].

ECT (Electric Convulsive Therapy) used to be prevalent within psychiatry but is today reserved mainly for deep depressions, particularly in melancholic and psychotic cases. In cer $\neg$ tain cases this treatment is administered against the will of the patient. Each individual treatment causes an amnesia that will flow together and form a blackout for the time the treatment occurred. Therefore ECT patients have unclear memory of the treatment, also long term. ECT has three effects.

i. Increase of dopamine receptor synthesis

ii. Heightened 5-HT receptor sensitivity

iii. Inhibited noradrenalin release

Supportive therapy, interpersonal therapy, and cognitive behavioural therapy are the three therapeutic methods employed for depression. Supportive therapy is often regarded with mistrust; however, after recovery it is often evident that it was the only method that helped the patient to keep up hope at the lowest point. Conflict processing psychotherapy is initiated when the somatic treatment is reduced or has clearly reduced the depressive signs, such as anti-aggression, excessive demands, misdirected intentions, or weak self-esteem. Social intervention and psychotherapy usually happen in parallel. Depressions are often caused by a disturbed relation to a partner, and therefore best results may be obtained in couple or family therapy where both parties can be present simultaneously compared to a situation where the therapist is working with only one of the partners at a time [2].

Cognitive behavioural therapy is a version of pedagogical psychotherapy. It is based upon the idea that cognitive factors - ways of thinking about oneself, the environment, and the future - has as impact on mental conditions, and that influencing thinking can have beneficial effects on mood [9]. Within CBT one also holds that influencing mood will have effects on temper as well. Thought templates are being construed during the process of upbringing and create patterns and interpret various events. Some templates may be negative, such as "I don't deserve to be loved", or "No one wants me". Such an individual may react to rejection with de-pression. Given such a background the risk for depression is great, that is, one will not register positive impacts, only the negative. Positive memories are shut up selectively and the individual is incapable of seeing his or her own part in the positive things that happen and assumes the blame for all negative things. In this way one gets stuck in the depression and tend to think in either-or categories - "I fail at everything I do".
One will come to general conclusions from one isolated negative event that confirms one single failure. Instead of an unbiased narrative of one's shortcomings one comes up with a label: "I am a failure". Eventually the depressive tirade will increase: "I am worthless, life is meaningless, and future is hope ᄀless" [2]. Interpersonal psychotherapy (IPT) is a synthesis of dynamic and cognitive psychotherapy. The purpose of IPT is modification of those distracting relations that may lead to depression, such as crises, family and relation conflicts, difficulties of accepting changes in life circum $\neg$ stances, or weak capability for long term bonding with the environment [2].

Individuals with least social functional disorders were the most inclined to respond to interpersonal psychotherapy while those with least cognitive dysfunctions were the most inclined to respond to cognitive psychotherapy (the least severe cases responded best). Individuals with the most reduced working capability, such as those with the highest symptom profile, were the most inclined to respond to imipramine [10]. How depression and mania periods are treated depends upon the severity of the symptoms. With lighter depressions conversational therapy may suffice. However, there is often a need for anti-depressive medication with depression and antimania medication for the various degrees of mania. The crucial point is to find a relevant mood stabiliser when consulting the doctor. It has been scientifically proven that lithium and various anti-epileptic medications will stabilise mood [12].

Mood stabilisers play a main part in the treatment of bipolar disease while anti-psychotic medication is used for treatment of schizophrenia [3]. According to ICD-10 a mania period has the following criteria:

a. A limited episode in time with abnormal and prolonged elevated, extrovert or ill-tempered mood that will last for at least one week (or less if hospitalisation is deemed necessary)

b. Elevated self-esteem or delusions of grandeur

c. Decreased need for sleep (e g refreshed after only three hours sleep)

d. More talkative or eager to talk than usual

e. Topical flight or experiencing that thoughts fly away

f. Distractibility (attention is all too easily distracted towards unimportant or irrelevant out $\urcorner$ side stimuli)

g. Increased goal oriented activity (socially, at work or school, sexually) or psychomotor agitation)

h. Exaggerated interest in pleasurable activities that may have embarrassing consequences later (such as uninhibited buying frenzy, adultery, indiscriminate investments [2].

In treatment of maniacal syndromes these options are available and may be used separately or in combination: 
i. Tranquilising neuroleptic drugs, such as chlorpromazine and levomepromazine

ii. Non-tranquilising neuroleptic drugs (haloperidol)

iii. ECT

iv. Expectant

v. Lithium salts

vi. Antiepileptic drugs

vii. Benzodiazepines, such as Diazepam [2].

The two pharmacological methods available are neuroleptic and specifically anti-mania drugs. Tranquilising neuroleptic drugs have side effects like drowsiness and will decrease hyperactivity but do not, apart from that, affect the maniacal symptoms in a significant way. For this reason sedative neuroleptic drugs are used as night time medication. Haloperidol has neuroleptic effects, i.e. it will decrease motor unrest, nervousness and rage and produce indifference before emotionally charged stimuli without any significant fatigue or drowsiness nor impact upon mental functions. In maniacal situations haloperidol will yield a rapid decrease of the symptoms. Maniacs treated with haloperidol behave, as far as one can see, in normal ways and are capable of suppressing their symptoms during a half an hour conversation with their doctor, but keep their poor self-control, decreased self-criticism and expansiveness. These symptoms are not decreased even when the dose is greatly increased [2].

\section{Objectives and Issues}

The objectives of this study are obtaining greater understanding of the phenomenon of manic depression as well as elucidating established diagnostics and treatment forms for manic de $\neg$ pression. The following issues are included:

i. How is bipolar disease expressed?

ii. How does the doctor go about making the diagnosis?

iii. Which treatment forms are in use, and which one works best?

iv. What can the patient do on his or her own?

\section{Approach}

\section{Selection}

Based upon the objective of the study I have chosen to interview a number of persons who work within psychiatric care institutions where manic depression is common. These individ $\neg$ uals are active in various roles, such as nurse, psychologist, and psychiatrist. Finding personnel working with the manic depression diagnosis was relatively easy. However, coming across people who actually were willing to participate in the study proved more difficult. Some were asked to participate but declined. In order to find relevant individuals I contacted people who were closely acquainted with the phenomenon, who in turn, could tip me off with contact channels to other persons I could consult.

This approach is known as snowballing. My former colleagues have also been able to provide me with valuable information. This ap $\urcorner$ proach can be compared to a selection where individuals are chosen independently from the population based upon certain characteristics (Olsson \& Sörensen, 2001). It is not intended here to reach any common ground or generalisations; the results are relevant for this study only. The literature has provided me with ideas for relevant questions, which hopefully would provide me with the answers, and further yield as much of a comprehensive picture as possible of the maniacal depressive phenomenon.

\section{Approach}

The choice of approach should be determined by the problem, and since the aim is to reach deeper understanding of the maniacal depressive phenomenon I believe a qualitative survey would be appropriate for interviews as a means to this end. The interviewees were six individuals of various ages and gender and were all contacted by telephone. They were informed about the objective and subject of the study and were thereafter asked whether they wished to participate. Time and place for the interviews were arranged. Three of the six interviews were performed at the interviewees' workplace; two were done by e-mail, and one by telephone. The conversations lasted about one to two hours. Two of the interviewees in Gothenburg did not attend the appointment and three were not qualified nurses (they said they had forgotten a good deal).

\section{Processing and Data Analysis}

When the data collection was completed I had a few pages to process. I started the analysis work by reading the entire interview material, and in an attempt to make some structure out of the sheer massiveness of it all I arranged the text in a temporal and social order and hoped it would reveal its meaning. As a first step I was looking for a number of themes that would comprise a starting point for the processing and analysis that lay ahead. I practical terms, working out a structure or looking for themes meant carefully reading each interview, sen $\neg$ tence by sentence thus being able to sort the contents into themes. The final structure ended up being comprised of the following themes: The experience of manic depression, diagnos $\neg$ tics and treatment of the phenomenon and what a patient may do by himself.

The second step consisted mainly of data crunching to find verbal expressions of relations of some kind. I was primarily looking for quotes where the interviewee expressed some relartion, i.e. something that had a relation to something else. During the entire analysis work I had feedback to the literature on manic depression. The results and the discussion are presented along the different themes mentioned earlier, and will start with the individual's own experiาence of manic depression. 


\section{Ethical Position}

Ethical judgement is an important part of research, since research "poses ethical problems on which most people don't need to take a position" (Pettersson, 1994). In research, norms and values are adhered to that are tied to the department that conducts the research.

Choice of subject as well as the broader consequences of the research are important factors to consider, according to Pettersson (1994). The interview material will be accessible to myself only and will be stored in a way that it can only be used in connection to this study.

\section{Validity and Reliability}

Since this study is qualitative I do not claim it has any generalising potential; I just wanted to understand and learn more about the phenomenon and the individual's experience of it. Validity means that I have surveyed that which the study set out to study (Kvale, 1997). The interviewees have furthermore been encouraged to express their views freely.

Good reliability means diligence and consistency. According to Pettersson (1994) the re $\neg$ searcher's own comprehension can have an impact upon the results, and therefore the researcher must be reviewed concerning printout, analysis, and reports, although the interviewees' accounts also must be questioned concerning their veracity. The analysis and reports have been conducted according to choice of method.

\section{Results and Discussion}

The ward personnel have been first hand witnesses to the various symptoms that patients with manic depression experienced in connection to their illness. The pathology of depression is this: Apathy, inhibition of gestures and speech, feelings of worthlessness, deep gloom, melan $\neg$ choly, feelings of guilt, meaninglessness, fear and suicidal thoughts, thoughts about death, procrastination, hypochondria, impotency, discomfort coming with early awakening, etc. Bodily sensations of discomfort may be vague: Weakness, powerlessness, general sensation of illness, or located in the chest, belly, or head area that is experienced as pressure, de-spondency, or tightness and hopelessness. There are also disorders of central functions present, such as disruption of sleep, appetite, eating, and sexuality. Non-commitment and indifference towards the environment have also been noticed.

Chief symptoms of mania are elation, aggression or irritability, increased energy and elevated activity levels (buying, working, and learning), elevated self-esteem, sometimes delusions, poor disease awareness, indiscriminate actions causing suffering, rushing thoughts, unusually strong extroversion, often also increased sexual interest, or risky behaviour. During the mania periods the pathology is the opposite of the depressive periods - one is infused with determination and zest for life. The informants had different opinions about the diagnosis and some of them maintained that the diagnosis should be based upon the symptoms that are present. Others were of the opinion that this should instead be deduced from clinical observation and the DSM-IV (Diagnostic Statistical Manual of Mental Disorders Fourth Edition) tool.

Psychiatrists, on the other hand, argued that the diagnosis should be derived from clinical observation, observations made by the environment and the criteria found in DSM-IV as well as by performing a thorough review of the medical history. Others again held that there is above all a need for specific attention when seeing patients that have experienced more than one extended and deeper depression episode: It is only when a mania relapse is obvious or when an attentive psychiatrist is doing an anamnesis that the bipolar nature of the disease becomes evident. The pathology with its extreme mood swings is so typical that the doctor or loved ones seldom have reason to doubt that the diagnosis is correct. In connection to the first episode there may of course be some uncertainties; it is however often helpful to learn whether there is a similar pathology found among the sufferer's close relatives.

The strategies employed are totally dependent upon how far into the treatment one has reached. If it is a matter of the first mania episode, then obviously, according to some informants, emergency medication that has immediate effect is necessary. If the mania wears off then one can have a strategy in common with the patient on how to prevent future mood swings by using mood regulators. It is important to understand that mood regulators do not provide $100 \%$ protection, and therefore it is equally important to work with the patient and make him an expert on his own disease. In that way one may prevent many relapses as well as taking action at a very early stage.

The strategies for choosing the treatment are as follows. During the emergency stage, fast and effective medication that suppresses the patient's maniacal condition, and later on, long term medication and mood stabilisers (lithium, antileptic drugs), and even later on, psychotherapy. For depression, anti-depressive medication is used, or even ECT. The medical treatment is effective in the sense that it works to stabilise the neurotransmitters that are involved. Another medical group hold that the strategies that should be employed during the emergency stage are the use of fast and effective anti-psychotic drugs to suppress the patient's maniacal condition, and then long term medication, such as mood stabilisers (lithium, antileptic drugs).

According to the informants, the methods of treating mania and depression periods are dependent on the severity of the symptoms. For lighter depressions conversational therapy can be sufficient. However often there is a need of anti-depressive drugs for depression and anti-maniacal medication at various degrees of mania. The important matter is finding a mood stabilising medication that works when consulting the doctor. It is scientifically proven that lithium and antileptic drugs stabilise 
mood. They have anti-mania effects and also keep the mood at reasonable levels thereby preventing that new relapses occur. There are, then, several choices to test before one finds the optional medication. Waking therapy is, according to the medical staff, a temporary method that is effective on melancholic depressions that do not work on other types of depression. The downside: short term effects and great risk of relapse.

Light therapy is an effective option for depressions that are connected to the dark season; the downside is that it also has short term effects. Some of the medical staff argued that lithium treatment is the best treatment for bipolar disease, while others argued that pharmacological treatment in conjunction with preventive mood stabilisers (lithium) and supportivetherapeutic treatment is best for bipolar disease, and other informants again held that it all depends upon the condition of the patient and his or her willingness to cooperate. The crucial step is motivating the patient to accept some kind of pharmacological treatment and make the patient carry it out to completion. There were persons who also believed that ECT is the best treatment, while others believed that to be erroneous.

Psychotherapy does, according to the medical staff, play an important part in the treatment of bipolar disease, particularly during its depressive stages. The psychotherapeutic methods employed for depression are cognitive behavioural psychotherapy, inter-personal therapy, and supportive psychotherapy. However, during the mania phases it is almost impossible to use psychotherapy because the patient is irritable, aggressive and finds it difficult to sit still.

According to some of the staff, what the patient can do by himself is follow the medication schedule, avoid experimenting, avoid challenging and stressful situations, plan both short and long term, sleep properly each night, avoid shift work, avoid leading positions, stay within harmonious environments, and work at places with reasonable performance requirements. What the patients can do, others said, was learning everything about their disease and the early warning signs since there usually is the same pattern at every relapse, and that it is important to communicate that information to loved ones and the medical staff, and seek help when the symptoms begin to be noticeable. The preventive method used for reducing the risk of relapse is lithium treatment. The medical staffs were consistent in their view that pharmacological treatment in conjunction with mood stabilisers (lithium) and supportive-therapeutic treatment is the best method for treating bipolar disease. With right treatment individuals with bipolar disease can lead a normal life.

\section{Concluding Discussion}

The objective of this study has been to obtain increased understanding of the maniacal depressive phenomenon from theoretical and practical perspectives, how it is diagnosed and treated, and what the patient can do him. Some view the mania period as a suppressed or denied depression. However, I do not share that view, because there are for example symptoms such as hyperphagia, weight gain and suicide that are common with both mania and depression periods; i.e., these characteristics are common with both young maniacal and depressive patients.

Bipolar disease is a fundamental and profound change of the basic mood in two opposite directions. Individuals experience that their mood disappears down into gloom, powerlessness, a black hole of brooding, guilt, meaninglessness, fear, and suicidal thoughts. Or that their mood is elevated with symptoms such as decreased need for sleep, and the experience of an unending flow of energy. Many on-going projects, rushing thoughts, extroversion that is not normal, often accompanied by heightened interest for sex or risky behaviour. Bigger expenses and indiscrimination often make this mood change produce great suffering, if not for the patient directly, then for people around the sufferer. According to the interviewees, manic depression is a life-long phenomenon, i.e., it has a tendency to recur starting with an intense sense of joy - mania, or gloom - depression.

The symptoms sometimes mark very com $\neg$ prehensive changes of personality. It seems to be a very special disease since it often has two different moods. Usually the mania phase starts with increased activity in combination with immense social candour. The maniacal patient is seen as a funny and talkative social individ $\neg$ ual of many ideas and devices. As opposed to the depressive patient the sexual drive increases at the same time as the need for sleep is decreased, and the need for action is increased along with increasing random activities around the clock [13]. During the mania period the patient is in danger of committing himself to criminal or socially deleterious circumstances. The disease may be explained from different angles, such heredity, physiologically or biologically, and psychosocial factors. Bipolar disease has a spectral diagnosis with many subheadings and is presently being reworked into a new classification system which in all probability will consist of at least four subcategories of bipolar diseases.

The diagnostics is not as straightforward as one may think. This is due to the simple fact that individuals with bipolar disease are more inclined to seek help during the depression phases than under the mania and hypo mania phases. This is, in turn because mania and hypomania are never mentioned spontaneously since it is so embarrassing, and especially hypo mania periods are not noticed as such by the patient but experienced more as just pleasurable hyperactivity. One may add to this that society with its Lutheran outlook on working capability will of course praise people who work and are sociable, hyper productive people who can do one thing or two. Therefore specific attention is above all required when seeing patients who have experienced more than one sustained and profound depression period; it is not until a mania relapse is obvious, or when a vigilant psychiatrist makes an anamnesis, that the bipolar nature becomes visible.

According to the medical staff, lithium prophylaxis is an important pillar in treating bipolar disease, and psychiatrists in Sweden and elsewhere can hardly count the patients on lithium 


\section{Psychology and Behavioral Science International Journal}

that never experienced a single depressive or mania relapse after they became positively inclined towards lithium medication. Lithium treatment causes the rate of thyroid to decrease making lithium a good option during the mania phase; however, its side effects during the depression phase can worsen the patient's condition. Added to that, there may be various reasons not to choose this medication as phase prophylaxis, but another medication instead, mostly because there may be other somatic diseases to consider, or that there may be intolerance against the medication itself. The best treatment, however, is a combination of working phase prophylaxis and one informed expert who is the patient self, and a sensitive environment or a supportive loved one who in case of emergency could respond and contact the specialist psychiatry at the earliest possible stage to catch any potential deterioration.

The best measure the patient can take is seeing him- or herself as a skilled and capable fellow human being. The disease implies no degradation but demands taking up the task of living with a specific vulnerability that can be met with adequate attention, a doable but often lifelong medication as well as sparse but much needed contact with psychiatric healthcare. Apart from that it certainly depends upon the individual's needs of seeing, not only a doctor but also a self-help group. Coming across an employer that shows understanding and support rehabilitation after a relapse and a partner who is expressing tolerance and initiative make good conditions for returning to a normal life. A significant problem, however, is that the overlapping of bipolar disease and substance abuse makes treatment, prognosis, and social life much more precarious.

In addition the medical staffs need excellent supervision and training. I believe in particular that supervision is perhaps the most important of all staff care measures. It can enhance understanding and respect among the work team and thereby becoming a strong instrument against the division that otherwise easily could destroy the therapeutic atmosphere at a ward. Training may provide as broad a common knowledge ground as possible, making work meaningful for all. The reality that is reflected today is that a larger and more visible group of individuals are suffering from affective syndromes, in particular the depressive syndromes that have been the most common syndrome among young people.

\section{Glossary}

AD: Anti-depressiva medication

Agitation: Restless movements

Aneuryism: Blood swelling of an artery

Anhedonia: Incapability of enjoyment and finding joy in life

Anterograde amnesia: Incapability of remembering things that were taught before the memory loss ocurred

Anticholinergical: Antispasmolytic with inhibiting effects on bowels

Bipolar disorder: Manic depression

Cerebral: Pertaining to cerebrum

Cycloid syndrome: A type of mental disorder that is a combination of affective syndrome and schizophrenia

Cyclothymia: Mental disorder that is a milder version of manic depression

DSM-IV: Diagnostic Statistical Manual of Mental disorders, Fourth Edition

Dysphoria: Gloom

Dysthymia: Distress, depression

ECT: Electric Convulsive Therapy (electrical shock therapy)

Epigast: Above belly

Eufori: $\quad$ Strong sense of happiness

Extacy: Intense experience of joy connected to the sense of being at one with God, nature or the universe

Gastrointestinal Pertaining to stomach and intestines

Hyperactivitet: Increased activity

Intracrainial: $\quad$ Located inside head Cardiovascular Pertaining to heart and blood vessels

Catatonic: A form of schizophrenia

Colinergic: Responding to acethylcholine lowering blood pressure

Counter indication: Sign poining in the opposite directio Retrograds amnesia: Incapability of remembering things that were taught after the memory loss ocurred

Serotonin: Transmitter substance in CNS (Central Nerve System)

Stupor: Complete immovability, total mental blockage

Tardive Developing slowly

Refractory: Insusceptible disease

Tremor: Trembling, shuddering

\section{References}

1. Palmgren L (1998) Att vårda våldsamma patienter. Stockholm: Natur och kultur.

2. Ottosson JO (2000) Psykiatri. Stockholm: Liber AB.

3. Terence A. Ketter, Po.W. Wang, Olga V. Becker, Cecylia Nowakowska, Yen-shou Yang (2003) Psychotic bipolar disorders: dimensionally similar to or categorically different from schizophrenia." Journal of psychiatric Research. Stanford 38(1): 47-61.

4. Goodwin FK, Ghaemi SN (2003) The course of bipolar disorder and the nature of agitated depression Am J Psychiarty 160(12): 2077-2079. 
5. Skärsäter I (2002) The importance of social support for men and women, suffering from major depression. A comperative and explorative study. Göteborg: Vasastadens Bokbinderi AB.

6. Benazzi F, Koukopoulos A, Akiskal HS (2004). Toward a validation of a new definition of agitated depression as a bipolar mixed state (mixed depression). Eur Psychiatry 19(2): 85-90.

7. Timothy E Wilens, Janet Wozniak (2003) Bipolar Disorders in Children and Adolescents:Diagnostic and Therapeutic Issues. Psychiatric Times 20: 8 .

8. Sheue Jane Hou, Feng Chang Yen, Chin Ya Cheng, Shinh Jen Tsai, Chen Jee, Hong (2004) X-bonding protein 1 (XBP1) C-1165 polymorphisms in bipolar disorders and age of onset. Neuroscience Letters 367. Taipe Veterans General Hospital. 232-234.
9. Jansson L (1998) Handbok i kognitiv terapi vid depression. Stockholm: Natur och Kultur.

10. Sotsky SM, Glass DR, Shea MT, Pilkonis PA, Collins JF, et al. (1991) Patient predictors of response to psychotherapy and pharmacotherapy: Finding in the NIMH treatment of depression collaborative research program. Am J Psychiatry 148(8): 997-1008.

11. Haugsgjerd S (1999) Psykiskt lidande. Stockholm: Natur och kultur.

12. Ågren H (2000) Bipolär sjukdom.

13. Cullberg J (2001) Dynamisk psykiatri. Stockholm: Natur och kultur.

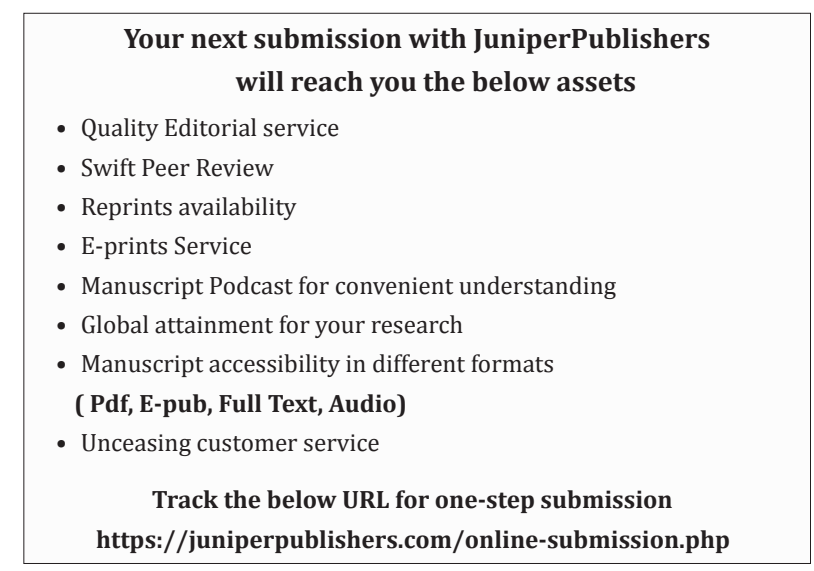

